\title{
THE GELFAND TRANSFORM OF HOMOGENEOUS DASTRIBUTIONS ON HEISENBERG TYPE GROUPS
}

\author{
FRANCESCA ASTENGO ${ }^{\varpi}$ and BIANCA DI BLASIO
}

(Received 17 January 2005; revised 19 May 2005)

Communicated by A. J. Pryde

\begin{abstract}
A distribution on a Heisenberg type group of homogeneous dimension $Q$ is a biradial kernel of type $\alpha$ if it coincides with a biradial function, homogeneous of degree $\alpha-Q$, and smooth away from the identity. We prove that a distribution is a biradial kernel of type $\alpha, 0 \leq \alpha<Q$, if and only if its Gelfand transform, defined on the Heisenberg fan, extends to a smooth even function on the upper half plane, homogeneous of degree $-\alpha / 2$. A similar result holds for radial kernels on the Heisenberg group.
\end{abstract}

2000 Mathematics subject classification: primary 43A80; secondary 22E30.

Keywords and phrases: Heisenberg type groups, homogeneous distributions.

\section{Introduction}

Homogeneous distributions play a fundamental rôle in harmonic analysis. It is wellknown that a distribution on $\mathbb{R}^{n}$ is homogeneous of degree $\alpha$ and smooth away from the origin if and only if its Fourier transform is a homogeneous distribution of degree $-n-\alpha$, and is smooth away from the origin. The aim of this paper is to obtain a similar characterisation in the Heisenberg group setting.

Let $N \simeq \mathbb{R}^{2 m} \times \mathbb{R}^{k}$ be a Heisenberg type group of homogeneous dimension $Q=$ $2 m+2 k$. We say that a function $f$ on $N$ is biradial if there exists a function $f_{0}$ on $\mathbb{R}^{2}$ such that $f(X, Z)=f_{0}(|X|,|Z|)$ for every $(X, Z)$ in $N$. A distribution on $N$ is a biradial kernel of type $\alpha$ if it coincides with a biradial homogeneous function of degree $\alpha-Q$, and smooth away from the identity of $N$.

In this paper we characterise the image under the Gelfand transform of biradial kernels of type $\alpha, 0 \leq \alpha<Q$. Such kernels are involved in many analytic problems.

Work partially supported by the Progetto Cofinanziato M.I.U.R. "Analisi Armonica".

(C) 2006 Australian Mathematical Society $1446-7887 / 06 \$ A 2.00+0.00$ 
Indeed, if $0 \leq \alpha<Q$ and $1 / p-1 / q=\alpha / Q$, then they are $L^{p}-L^{q}$ convolutors. Moreover, they appear in the functional calculus of many operators, such as the Heisenberg sublaplacian [11] or the Hodge Laplacian [10]. Other results concerning homogeneous distributions on the Heisenberg group can be found in $[3,4,6-8,12]$.

To describe the results, we introduce some notation. Let $\mathbb{R}^{*}=\mathbb{R} \backslash\{0\}, \mathbb{R}^{+}=$ $(0,+\infty), \mathbb{R}_{+}^{2}=\left\{(\lambda, \xi) \in \mathbb{R}^{2}: \xi>0\right\}$, and $\mathbb{N}=\{0,1,2, \ldots\}$.

The convolution algebra of integrable biradial functions on $N$ is a commutative Banach algebra and its Gelfand spectrum can be be parametrised by a pair $(\lambda, d)$, where $\lambda$ is in $\mathbb{R}^{+}$and $d$ is in $\mathbb{N}$, and by a nonnegative real number $\xi$. Since the subset $\{\xi \geq 0\}$ of the Gelfand spectrum has zero Plancherel measure, it will be usually disregarded.

It is easy to see that if $K$ is a biradial kernel of type $\alpha$, then its Gelfand transform $\widehat{K}$ satisfies

$$
\widehat{K}(\lambda, d)=\lambda^{-\alpha / 2} \widehat{K}(1, d) \text { for all }(\lambda, d) \in \mathbb{B}^{+} \times \mathbb{N} .
$$

Geller [8, Theorem 2.7, Theorem 3.7] characterised the image under the Gelfand transform of biradial kernels of type $\alpha$ on the Heisenberg group in terms of the behaviour at infinity of the sequences $d \mapsto \widehat{K}(1, d)$. He actually proved this kind of result for general (not necessarily biradial) kernels on the Heisenberg group. In this paper we continue this investigation.

If $K$ is a biradial kernel of type $\alpha$, we can extend $\widehat{K}(\lambda, d)$ to negative values of $\lambda$ by

$$
\widehat{K}(\lambda, d)=\widehat{K}(-\lambda, d) \text { for all }(\lambda, d) \in \mathbb{R}^{*} \times \mathbb{N},
$$

and we can consider $\widehat{K}$ as a function defined on the so-called Heisenberg fan, that is, the subset of $\mathbb{R}^{2}$ given by

$$
\left(\bigcup_{d \in \mathbb{N}}\left\{(\lambda, \xi) \in \mathbb{R}^{2}: \xi=|\lambda|(2 d+m), \lambda \neq 0\right\}\right) \cup\left\{(0, \xi) \in \mathbb{R}^{2}: \xi \geq 0\right\} .
$$

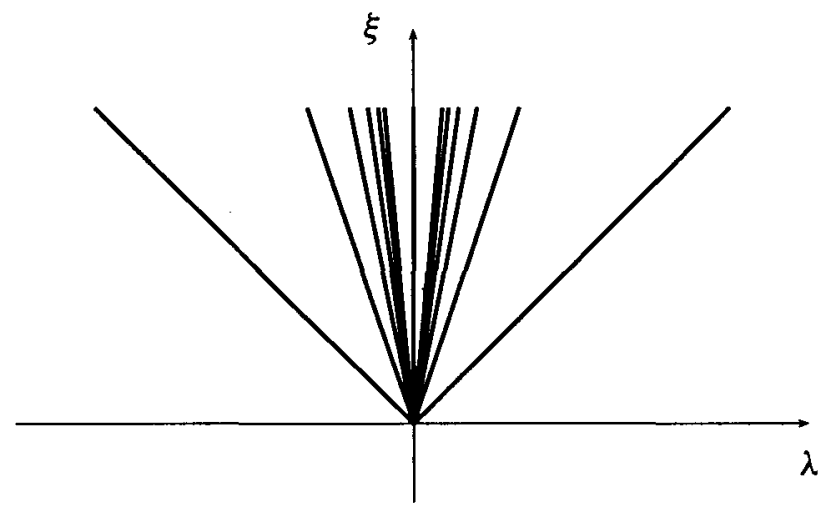


We prove that a function $\psi: \mathbb{R}^{*} \times \mathbb{N} \rightarrow \mathbb{C}$ is the Gelfand transform of a biradial kernel of type $\alpha$ if and only if it is the restriction to the Heisenberg fan of a smooth function on $\mathbb{R}_{+}^{2}$, homogeneous of degree $-\alpha / 2$, and even in the first variable.

When $\alpha=0$, we construct the extension $F$ as follows. Inspired by the work of Müller, Ricci, and Stein [11], we exploit the Poisson summation formula: if $\varphi$ is a Schwartz function on the real line whose Fourier transform $\mathscr{F} \varphi$ has compact support in the interval $[-3 / 4,3 / 4]$ and such that $\sum_{j \in \mathbb{Z}} \mathscr{F} \varphi(x+j)=1$ for any real $x$, then $\varphi(0)=1$ and $\varphi(j)=0$ for $j$ in $\mathbb{Z} \backslash\{0\}$. If $K$ is a biradial kernel of type 0 , we extend in a suitable way the sequences $(\widehat{K}( \pm 1, d))_{d \in \mathbb{N}}$ to $\mathbb{Z}$, and we define the function $F$ on the upper half plane by the formula

$$
F(\lambda, \xi)= \begin{cases}\sum_{j \in \mathbb{Z}} \widehat{K}(\lambda, j) \varphi\left(\frac{1}{2}\left(\frac{\xi}{|\lambda|}-m\right)-j\right) & \text { for all }(\lambda, \xi) \in \mathbb{R}^{*} \times \mathbb{R}^{+} \\ \widehat{K}(\xi) & \text { for all }(\lambda, \xi) \in\{0\} \times \mathbb{R}^{+}\end{cases}
$$

It easy to see that $F$ is smooth on $\mathbb{R}^{*} \times \mathbb{R}^{+}$and that $F(\lambda,|\lambda|(2 d+m))=\widehat{K}(\lambda, d)$, when $\lambda$ is in $\mathbb{R}^{*}$ and $d$ is in $\mathbb{N}$. The most elaborate part is the proof of the smoothness of the function $F$ on the $\xi$-axis; for this it is necessary to know the asymptotic expansion at infinity of the sequences $(\widehat{K}( \pm 1, d))_{d \in \mathbb{N}}$ (see [8, Theorem 2.7, Theorem 3.7] for the case where $N$ is the Heisenberg group). The case of biradial kernels of type $\alpha$, where $0<\alpha<Q$, may be deduced from the case where $\alpha=0$.

We also consider the case of radial (not necessarily biradial) kernels on the Heisenberg group. We first prove our main result in this case (Theorem 6.1), and with minor modifications deduce the result for biradial kernels on Heisenberg type groups (Theorem 3.2).

Our paper is organised as follows. Section 2 contains preliminary material on Heisenberg type groups, in particular on the Gelfand transform of biradial functions. In Section 3 we describe our result for biradial kernels on Heisenberg type groups. Section 4 is self-contained and deals with the problem of extending a sequence in a smooth way and preserving its asymptotic expansion at infinity. The proof of our results is contained in Sections 5 and 6.

It is a pleasure to thank Giancarlo Mauceri and Fulvio Ricci for many helpful conversations on the subject of this paper.

\section{Heisenberg type groups and the Gelfand transform}

2.1. Heisenberg type groups Let $\boldsymbol{n}$ be a two-step real nilpotent Lie algebra endowed with an inner product $\langle,\rangle_{\mathfrak{n}}$. Write $\mathfrak{n}$ as an orthogonal sum $\mathfrak{n}=\mathfrak{v} \oplus \mathfrak{z}$, where $\mathfrak{z}$ is the center of $\mathbf{n}$.

For each $Z$ in $\mathfrak{z}$, define the map $J_{Z}: \mathfrak{v} \rightarrow \mathfrak{v}$ by the formula

$$
\left\langle J_{Z} X, Y\right\rangle_{\mathfrak{n}}=\langle[X, Y], Z\rangle_{\mathfrak{n}} \text { for all } X, Y \in \mathfrak{v} .
$$


According to Kaplan [9], the Lie algebra $\mathfrak{n}$ is said to be $H$-type if, for every $Z$ in $\mathfrak{z}$,

$$
J_{Z}^{2}=-|Z|^{2} I_{\mathfrak{v}}
$$

where $I_{\mathfrak{v}}$ is the identity on $\mathfrak{v}$. A connected and simply connected Lie group $N$, whose Lie algebra is an $H$-type algebra, is said to be a Heisenberg type group, or $H$-type group for short.

Since $\mathfrak{n}$ is a nilpotent Lie algebra, the exponential map is surjective. We can then parametrise the elements of $N=\exp \mathfrak{n}$ by $(X, Z)$, for $X$ in $\mathfrak{v}$ and $Z$ in $\mathfrak{z}$. By the Baker-Campbell-Hausdorff formula it follows that the product law in $N$ is

$$
(X, Z)\left(X^{\prime}, Z^{\prime}\right)=\left(X+X^{\prime}, Z+Z^{\prime}+\left[X, X^{\prime}\right] / 2\right)
$$

for all $X, X^{\prime} \in \mathfrak{v}$ and $Z, Z^{\prime} \in \mathfrak{z}$. We denote by $d X$ and $d Z$ the Lebesgue measures on $\mathfrak{v}$ and on $\mathfrak{z}$ respectively; it is easy to check that $d n=d X d Z$ is a Haar measure on $N$. For every unit vector $Z$ in $\mathfrak{z}$, the map $J_{Z}$ defines a complex structure on $\mathfrak{v}$; therefore $\mathfrak{v}$ has even dimension, say $2 \mathrm{~m}$. We denote the dimension of the center $\mathfrak{z}$ by $k$, and by $Q=2 m+2 k$ the homogeneous dimension of $N$, with respect to the anisotropic dilations

$$
D_{r}(X, Z)=\left(r X, r^{2} Z\right) \text { for all }(X, Z) \in N \text { and } r>0 .
$$

We fix orthonormal bases $\left\{E_{j}\right\}_{j=1}^{2 m}$ of $\mathfrak{v}$ and $\left\{U_{j}\right\}_{j=1}^{k}$ of $\mathfrak{z}$. Given $V$ in $\mathfrak{n}$, we also write $V$ for the associated left-invariant vector field, that is,

$$
V f(n)=\left.\frac{d}{d t} f(n \exp (t V))\right|_{t=0} \text { for all } n \in N \text { and } f \in C^{\infty}(N) .
$$

Define the sublaplacians $\mathscr{L}_{1}$ and $\mathscr{L}_{2}$ on $N$ by

$$
\mathscr{L}_{1}=-\sum_{j=1}^{2 m} E_{j}^{2}, \quad \text { and } \quad \mathscr{L}_{2}=-\sum_{j=1}^{k} U_{j}^{2}
$$

2.2. The Gelfand transform for biradial functions We say that a function $f$ on $N$ is biradial if there exists a function $f_{0}$ on $\mathbb{R}^{2}$ such that $f(X, Z)=f_{0}(|X|,|Z|)$ for every $(X, Z)$ in $N$. We recall some facts from $[1,2]$ regarding the Gelfand spectrum $\mathscr{G}$ of the commutative algebra of biradial integrable functions. It is known that $\mathscr{G}$ is the set of bounded spherical functions on $N$. These are given by the rules

$$
\begin{aligned}
\phi_{\lambda, d}(X, Z) & =e^{-\lambda|X|^{2} / 4} \frac{L_{d}^{m-1}\left(\lambda|X|^{2} / 2\right)}{\left(\begin{array}{c}
d+m-1 \\
d
\end{array}\right)} \int_{S_{\mathfrak{z}}} e^{i \lambda\left(Z, Z^{\prime}\right)} d \mu_{\mathfrak{z}}\left(Z^{\prime}\right), \\
\phi_{\xi}(X, Z) & =\int_{S_{\mathfrak{v}}} e^{i \sqrt{\xi}\left(X, X^{\prime}\right)} d \mu_{\mathfrak{v}}\left(X^{\prime}\right),
\end{aligned}
$$


for every $(X, Z)$ in $N, \lambda>0, d \in \mathbb{N}$, and $\xi \geq 0$. As usual, $L_{d}^{\beta}$ denotes the $d^{\text {th }}$ Laguerre polynomial of order $\beta$, that is,

$$
L_{d}^{\beta}(u)=\sum_{j=0}^{d}\left(\begin{array}{l}
d+\beta \\
d-j
\end{array}\right) \frac{(-u)^{j}}{j !} \quad \text { for all } u \in \mathbb{R} .
$$

Moreover, $\mu_{\mathfrak{v}}$ and $\mu_{\mathfrak{z}}$ are the surface measures of the unit spheres $S_{\mathfrak{v}}$ and $S_{\mathfrak{z}}$ in $\mathfrak{v}$ and $z$ respectively, normalized to have mass 1 . Therefore $\mathscr{G}$ can be parametrized by $\left(\mathbb{R}^{+} \times \mathbb{N}\right) \cup[0,+\infty)$. Denote by $\widehat{\mathscr{L}}_{j}(\phi)$ the eigenvalue of the spherical function $\phi$ with respect to $\mathscr{L}_{j}, j=1,2$. We recall that $\widehat{\mathscr{L}}_{1}\left(\phi_{\lambda, d}\right)=\lambda(2 d+m), \widehat{\mathscr{L}}_{2}\left(\phi_{\lambda, d}\right)=\lambda^{2}$, $\widehat{\mathscr{L}}_{1}\left(\phi_{\xi}\right)=\xi$, and $\widehat{\mathscr{L}}_{2}\left(\phi_{\xi}\right)=0$. Define a metric on $\mathscr{G}$ by

$$
d\left(\phi, \phi^{\prime}\right)=\left|\widehat{\mathscr{L}}_{1}(\phi)-\widehat{\mathscr{L}}_{1}\left(\phi^{\prime}\right)\right|+\left|\widehat{\mathscr{L}}_{2}(\phi)-\widehat{\mathscr{L}}_{2}\left(\phi^{\prime}\right)\right| .
$$

Then this metric induces on the Gelfand spectrum $\mathscr{G}$ the topology of uniform convergence on compact sets.

We define the Gelfand transform $\widehat{f}$ of a biradial integrable function $f$ on $N$ by the rule

$$
\widehat{f}(\phi)=\int_{N} f(n) \overline{\phi(n)} d n \text { for all } \phi \in \mathscr{G} .
$$

For the sake of brevity, we shall often identify a spherical function $\phi_{\lambda, d}$ with the pair $(\lambda, d)$ in $\mathbb{R}^{+} \times \mathbb{N}$ and $\phi_{\xi}$ with the number $\xi$ in $[0,+\infty)$. According to this identification we shall write $\widehat{f}(\lambda, d)$ and $\widehat{f}(\xi)$ instead of $\widehat{f}\left(\phi_{\lambda, d}\right)$ and $\widehat{f}\left(\phi_{\xi}\right)$ respectively.

Let $\mathscr{D}_{+}$and $\mathscr{D}_{-}$be the operators defined by

$$
\begin{aligned}
& \mathscr{D}_{+} \psi(\lambda, d)=(d+m)(\psi(\lambda, d+1)-\psi(\lambda, d)), \\
& \mathscr{D}_{-} \psi(\lambda, d)=d(\psi(\lambda, d)-\psi(\lambda, d-1))
\end{aligned}
$$

for every $(\lambda, d)$ in $\mathbb{R}^{+} \times \mathbb{N}$ and $\psi: \mathbb{R}^{+} \times \mathbb{N} \rightarrow \mathbb{C}$. Moreover, let $M_{+}$and $M_{-}$be the operators defined by $M_{+}=\partial_{\lambda}-\lambda^{-1} \mathscr{D}_{+}$, and $M_{-}=\partial_{\lambda}-\lambda^{-1} \mathscr{D}_{-}$.

\section{Homogeneous distributions on Heisenberg type groups}

Let $\alpha$ be in $[0, Q)$. We say that a distribution $K$ on $N$ is a biradial kernel of type $\alpha$ if $\left\langle K, f \circ D_{r}\right\rangle=r^{-\alpha}\langle K, f\rangle$ for all $f \in C_{c}^{\infty}(N)$, and the kernel $K$ coincides with a smooth biradial function $f_{K}$ on $N \backslash\{(0,0)\}$. Then the function $f_{K}$ is homogeneous of degree $\alpha-Q$ and, when $\alpha=0$, it satisfies (see [5])

$$
\int_{s(0,1)} f_{K} d \sigma=0
$$


where $S(0,1)=\left\{(X, Z) \in N:|X|^{4} / 16+|Z|^{2}=1\right\}$ and $d \sigma$ is the surface measure induced by the Euclidean measure. Moreover, there exists a constant $c_{K}$ such that $K=$ p.v. $\left(f_{K}\right)+c_{K} \delta$, where p.v. denotes the principal value and $\delta$ the Dirac mass at the identity.

It is easy to check that a biradial kernel of type $\alpha$ is a tempered distribution. Moreover, if $K$ is a biradial kernel of type $\alpha$, then its Gelfand transform satisfies $\widehat{K}(\lambda, d)=\lambda^{-\alpha / 2} \widehat{K}(1, d)$ for all $d \in \mathbb{N}$, and almost every $\lambda \in \mathbb{R}^{+}$.

THEOREM 3.1. Suppose that $\alpha$ is in $[0, Q)$ and $\psi: \mathbb{B}^{+} \times \mathbb{N} \rightarrow \mathbb{C}$. The following conditions are equivalent:

(a) the function $\psi$ is the Gelfand transform of a biradial kernel of type $\alpha$ on $N$;

(b) for almost every $(\lambda, d)$ in $\mathbb{R}^{+} \times \mathbb{N}, \psi(\lambda, d)=\lambda^{-\alpha / 2} \psi(1, d)$, and there exists $a$ sequence $\left(c_{2 j}\right)$ in $\mathbb{C}$ such that for every $M \geq 0$

$$
\psi(1, d)=\sum_{j=0}^{M} c_{2 j}(2 d+m)^{-\alpha / 2-2 j}+o\left(d^{-2 M-\alpha / 2}\right), \quad d \rightarrow+\infty .
$$

This theorem has been proved by Geller [8, Theorem 2.7, Theorem 3.5] in the case where $N$ is the Heisenberg group and the homogeneous distributions are not necessarily biradial. The proof of Theorem 3.1 is given in Section 5: (a) implies (b) is proved in Lemma 5.1, Proposition 5.2, and Corollary 5.3, while (b) implies (a) follows by Theorem 3.2 .

We say that a function $F: \mathbb{R}_{+}^{2} \rightarrow \mathbb{C}$ is even if $F(-\lambda, \xi)=F(\lambda, \xi)$, for every $\lambda$ in $\mathbb{R}$ and $\xi>0$. We propose the following characterisation.

THEOREM 3.2. Suppose that $\alpha$ is in $[0, Q)$ and $\psi: \mathbb{R}^{+} \times \mathbb{N} \rightarrow \mathbb{C}$. The following conditions are equivalent:

(a) the function $\psi$ is the Gelfand transform of a biradial kernel of type $\alpha$ on $N$;

(c) there exists an even smooth function $F$ on $\mathbb{R}_{+}^{2}$, homogeneous of degree $-\alpha / 2$ such that $F(\lambda, \lambda(2 d+m))=\psi(\lambda, d)$ for almost every $(\lambda, d)$ in $\mathbb{R}^{+} \times \mathbb{N}$.

The proof of this theorem is postponed to Section 6 . There we actually consider the case where $N$ is the Heisenberg group and the homogeneous distributions are radial. Our proof can be adapted to biradial kernels on Heisenberg type groups without substantial changes. The critical point is the smoothness of the extension $F$ on the positive $\xi$-axis; for the proof of this fact we shall use the results contained in Section 4.

\section{Some asymptotic expansions}

In this section we explain how to interpolate a sequence in a smooth way, preserving its asymptotic expansion at infinity. We believe that it should be possible to find in the 
literature some of the arguments treated in this section. However we could not find a reference and, in order to make this paper self-contained, we include here most of the proofs of the results needed in the sequel.

4.1. Discrete asymptotic expansions Let $a=(a(j))_{j \in \mathbb{Z}}$ be a two-sided sequence of complex numbers. Denote by $\Delta$ the finite difference operator acting on the sequence $a$ by the rule $\Delta a(j)=a(j+1)-a(j)$ for all $j \in \mathbb{Z}$, and by $\tau_{h}$ the operator of translation by the integer $h$, that is, $\tau_{h} a(j)=a(j+h)$ for all $j \in \mathbb{Z}$. For any $a, b$ sequences, the following Leibniz rule holds

$$
\Delta(a b)(j)=\tau_{1} a(j) \Delta b(j)+\Delta a(j) b(j) \text { for all } j \in \mathbb{Z} .
$$

For every fixed $h$ in $\mathbb{Z} \backslash\{0\}$, let $b_{h}$ be the sequence defined by

$$
b_{h}(j)=\frac{1}{h}-\frac{1}{j} \text { for all } j \in \mathbb{Z} \backslash\{0\} .
$$

LEMMA 4.1. Suppose that $h$ is in $\mathbb{Z} \backslash\{0\}$. Then for every $n=1,2, \ldots$

$$
\sum_{d=0}^{n-1}\left(\tau_{d} \Delta b_{h}\right)(j)=\frac{n}{j(j+n)} \quad \text { for all } j \in \mathbb{Z} \backslash\{0,-1, \ldots,-n\} .
$$

Moreover, for every sequence $a=(a(j))_{j \in \mathbb{Z}}$,

$$
\Delta\left(\prod_{p=0}^{n} \tau_{p} a\right)(j)=\prod_{p=1}^{n} \tau_{p} a(j)\left(\sum_{q=0}^{n} \Delta \tau_{q} a\right)(j) \quad \text { for all } j \in \mathbb{Z} .
$$

ProOF. The first identity is true when $n=1$. Let $n \geq 2$ and suppose that the first identity holds true when $n=k-1$. If $j$ is in $\mathbb{Z} \backslash\{0,-1, \ldots,-k\}$, then, when $n=k$,

$$
\begin{aligned}
\sum_{d=0}^{k-1}\left(\tau_{d} \Delta b_{h}\right)(j) & =\sum_{d=0}^{k-2}\left(\tau_{d} \Delta b_{h}\right)(j)+\left(\tau_{k-1} \Delta b_{h}\right)(j) \\
& =\frac{k-1}{j(j+k-1)}+b_{h}(j+k)-b_{h}(j+k-1) \\
& =\frac{k}{j(j+k)} .
\end{aligned}
$$

The second property is true when $n=1$, because

$$
\Delta\left(a \tau_{1} a\right)(j)=a(j+1)[\Delta a(j)+\Delta a(j+1)] .
$$


Let $n \geq 2$, and suppose it holds true when $n=1, \ldots, k-1$. Then by the discrete Leibniz rule, when $n=k$,

$$
\begin{aligned}
\Delta\left(\prod_{p=0}^{k} \tau_{p} a\right)(j) & =\Delta\left(\prod_{p=0}^{k-1} \tau_{p} a \cdot \tau_{k} a\right)(j) \\
& =\tau_{1}\left(\prod_{p=0}^{k-1} \tau_{p} a\right)(j) \Delta \tau_{k} a(j)+\Delta\left(\prod_{p=0}^{k-1} \tau_{p} a\right)(j)\left(\tau_{k} a\right)(j) \\
& =\left(\prod_{p=1}^{k} \tau_{p} a\right)(j) \Delta \tau_{k} a(j)+\prod_{p=1}^{k-1} \tau_{p} a(j)\left(\sum_{q=0}^{k-1} \Delta \tau_{q} a\right)(j)\left(\tau_{k} a\right)(j) \\
& =\left(\prod_{p=1}^{k} \tau_{p} a\right)(j)\left(\sum_{q=0}^{k} \Delta \tau_{q} a\right)(j),
\end{aligned}
$$

as required.

For every $n$ in $\mathbb{N}$, let $T_{n}$ denote the operator defined by the recurrence relation:

$$
\begin{aligned}
& T_{0} a(j)=a(j) \text { for all } j \in \mathbb{Z}, \\
& T_{n} a(j)=\frac{j(j+n)}{n} \Delta T_{n-1} a(j) \quad n \geq 1 \text { and for all } j \in \mathbb{Z} .
\end{aligned}
$$

THEOREM 4.2. Suppose that $a=(a(j))_{j \in \mathbb{Z}}$ is a sequence of complex numbers. If the limits $\lim _{j \rightarrow \infty} T_{n} a(j)=(-1)^{n} \gamma_{n}, n \in \mathbb{N}$, exist and are finite, then, for every $n$ in $\mathbb{N}$,

$$
a(j)=\gamma_{0}+\frac{\gamma_{1}}{j}+\cdots+\frac{\gamma_{n}}{j^{n}}+o\left(|j|^{-n}\right) \quad|j| \rightarrow+\infty .
$$

PROOF. The case where $n=0$ is trivial, therefore we may suppose that $n \geq 1$. By the discrete Leibniz rule and evaluating the sum of a telescopic series, we obtain when $h$ is a fixed nonnegative integer

$$
\begin{aligned}
a(h) & =\gamma_{0}-\sum_{j=h}^{+\infty} \Delta a(j)=\gamma_{0}-\sum_{j=h}^{+\infty} \Delta b_{h}(j) T_{1} a(j) \\
& =\gamma_{0}-\sum_{j=h}^{+\infty} \Delta\left(b_{h} T_{1} a\right)(j)+\sum_{j=h}^{+\infty} b_{h}(j+1) \Delta T_{1} a(j) \\
& =\gamma_{0}+\frac{\gamma_{1}}{h}+\sum_{j=h}^{+\infty} b_{h}(j+1) \Delta T_{1} a(j) .
\end{aligned}
$$


When $n \geq 1$, define the sequence $r_{n}$ by

$$
r_{n}(h)=(-1)^{n+1} \sum_{j=h}^{+\infty} b_{h}(j+1) b_{h}(j+2) \cdots b_{h}(j+n) \Delta T_{n} a(j) \text { for all } h \geq 0 .
$$

Then $r_{n}(h)=o\left(h^{-n}\right)$ when $h \rightarrow+\infty$, because

$$
\begin{aligned}
\left|r_{n}(h)\right| & \leq \sum_{j=h}^{+\infty} \frac{1}{h^{n}}\left|\Delta T_{n} a(j)\right|=\sum_{j=h}^{+\infty} \frac{1}{h^{n}} \frac{n}{j(j+n)}\left|T_{n+1} a(j)\right| \\
& \leq C \frac{1}{h^{n}} \sum_{j=h}^{+\infty} \frac{1}{j(j+1)}=C \frac{1}{h^{n+1}} .
\end{aligned}
$$

We prove by induction that

$$
a(h)=\gamma_{0}+\frac{\gamma_{1}}{h}+\frac{\gamma_{2}}{h^{2}}+\cdots+\frac{\gamma_{n}}{h^{n}}+r_{n}(h) .
$$

We have just proved that this formula holds when $n=1$. Suppose it holds when $n=k-1$. Remember that by Lemma 4.1 we have

$$
T_{k} a(j)=\left[\sum_{d=0}^{k-1} \Delta \tau_{d} b_{h}(j)\right]^{-1} \Delta T_{k-1} a(j),
$$

so that

$$
\begin{aligned}
r_{k-1}(h) & =(-1)^{k} \sum_{j=h}^{+\infty} b_{h}(j+1) b_{h}(j+2) \cdots b_{h}(j+n-1) \Delta T_{k-1} a(j) \\
& =(-1)^{k} \sum_{j=h}^{+\infty} \prod_{d=1}^{k-1} \tau_{d} b_{h}(j) \sum_{d=0}^{k-1} \Delta \tau_{d} b_{h}(j)\left[\sum_{d=0}^{k-1} \Delta \tau_{d} b_{h}(j)\right]^{-1} \Delta T_{k-1} a(j) \\
& =(-1)^{k} \sum_{j=k}^{i-\infty} \Delta\left(\prod_{d=0}^{k-1} \tau_{d} b_{h}\right)(j) T_{k} a(j) \\
& =(-1)^{k} \sum_{j=h}^{+\infty} \Delta\left(\prod_{d=0}^{k-1} \tau_{d} b_{h} T_{k} a\right)(j)-(-1)^{k} \sum_{j=h}^{+\infty} \prod_{d=0}^{k-1} \tau_{d} b_{h}(j+1) \Delta T_{k} a(j) \\
& =\frac{\gamma_{k}}{h^{k}}+(-1)^{k+1} \sum_{j=h}^{+\infty} \prod_{d=1}^{k} \tau_{d} b_{h}(j) \Delta T_{k} a(j) \\
& =\frac{\gamma_{k}}{h^{k}}+r_{k}(h),
\end{aligned}
$$

that is, equation (2) holds when $n=k$, as required. The case $h \rightarrow-\infty$ can be treated in a similar way. 
4.2. From discrete to continuous asymptotic expansions In this subsection we make repeated use of the Poisson summation formula

$$
\sum_{j \in \mathbb{Z}} f(t+j) e^{-2 \pi i \xi(t+j)}=\sum_{j \in \mathbb{Z}} \mathscr{F} f(\xi+j) e^{2 \pi i j t} \quad \text { for all } t, \xi \in \mathbb{R},
$$

where $f$ is an integrable function on $\mathbb{R}$ with Fourier transform

$$
\mathscr{F} f(\xi)=\int_{\mathbb{R}} f(t) e^{-2 \pi i t \xi} d t \text { for all } \xi \in \mathbb{R} .
$$

Fix a Schwartz function $\varphi$ whose Fourier transform has compact support in $[-3 / 4,3 / 4]$, and such that $\sum_{j \in \mathbb{Z}} \mathscr{F} \varphi(t+j)=1$ for all $t \in \mathbb{R}$. By applying the Poisson summation formula to the function $\mathscr{F} \varphi$ we obtain

$$
\left.\sum_{j \in \mathbb{Z}} \varphi(\xi+j)=1 \quad \text { (for all } \xi \in \mathbb{R}\right), \quad \varphi(j)= \begin{cases}1 & \text { if } j=0, \\ 0 & \text { if } j \in \mathbb{Z} \backslash\{0\} .\end{cases}
$$

Suppose that $(a(j))_{j \in \mathbb{Z}}$ is a bounded sequence of complex numbers, and let $h=0,1$. Inspired by [11], we define the function $A_{h}$ on $\mathbb{R}$ by the rule

$$
A_{h}(t)=\sum_{j \in \mathbb{Z}} a(j) \varphi(t-(j+h / 2)) \quad \text { for all } t \in \mathbb{R} .
$$

Then, by the above mentioned properties, the function $A_{h}$ interpolates the sequence $a$, that is, $A_{h}(j+h / 2)=a(j)$ for every integer $j$. Being the convolution of a Schwartz function $\varphi$ and a tempered distribution supported on the integers or the half-integers, the functions $A_{0}$ and $A_{1}$ are smooth (and slowly increasing), and for every $n$ in $\mathbb{N}$

$$
A_{h}^{(n)}(t)=\sum_{j \in \mathbb{Z}} a(j) \varphi^{(n)}(t-(j+h / 2)) \quad \text { for all } t \in \mathbb{R}, h=0,1 .
$$

Let $\Theta$ denote the differential operator defined by

$$
\Theta f(t)=t^{2} f^{\prime}(t) \text { for all } t \in \mathbb{R} \text { and } f \in C^{\infty}(\mathbb{R}) .
$$

THEOREM 4.3. Let $h$ be in $\{0,1\}$. Suppose that the sequence $(a(j))_{j \in \mathbb{Z}}$ has the asymptotic expansion at infinity

$$
a(j)=\gamma_{0}+\frac{\gamma_{1}}{j+h / 2}+\cdots+\frac{\gamma_{n}}{(j+h / 2)^{n}}+o\left(|j|^{-n}\right), \quad|j| \rightarrow+\infty,
$$

and define the function $A_{h}$ on $\mathbb{R}$ as above. Then $A_{h}$ is smooth, the following limits exist and

$$
\lim _{|\ell| \rightarrow \infty}\left(\Theta^{n} A_{h}\right)(t)=(-1)^{n} n ! \gamma_{n} \quad \text { for all } n \in \mathbb{N}
$$


Note that (6) is equivalent to the fact that $A_{h}$ has the asymptotic expansion at infinity

$$
A_{h}(t)=\gamma_{0}+\frac{\gamma_{1}}{t}+\frac{\gamma_{2}}{t^{2}}+\cdots+\frac{\gamma_{n}}{t^{n}}+o\left(|t|^{-n}\right), \quad|t| \rightarrow+\infty .
$$

The proof of Theorem 4.3 is based on the following four lemmata.

LEMMA 4.4. Suppose that $b=(b(j))_{j \in \mathbb{Z}}$ is a sequence of complex numbers, vanishing at infinity and $\Phi$ is any Schwartz function on $\mathbb{R}$. Then

$$
\lim _{|t| \rightarrow+\infty} \sum_{j \in \mathbb{Z}} b(j) \Phi(t-j)=0 .
$$

ProOF. Let $\varepsilon>0$. Since $b$ vanishes at infinity, there exists a $J_{\varepsilon}>0$ such that $|b(j)|<\varepsilon$ for any $j$ such that $|j| \geq J_{\varepsilon}$. Since $\Phi$ is a Schwartz function, there exists a constant $C>0$ such that $|\Phi(t)| \leq C /(1+|t|)^{3}$ for every $t$ in $\mathbb{R}$. Hence, for every $t$ such that $|t|>1 / \varepsilon$, we conclude that $|\Phi(t)|<\varepsilon C /(1+|t|)^{2}$. Now we divide the sum into two parts and evaluate each part separately. Let $|t|>1 / \varepsilon+J_{\varepsilon}$. Note that, if $|j|<J_{\varepsilon}$, then $|t-j| \geq|t|-|j|>1 / \varepsilon$. Therefore,

$$
\begin{aligned}
\left|\sum_{j \in \mathbb{Z}} b(j) \Phi(t-j)\right| & \leq\left|\sum_{|j| \geq J_{\varepsilon}} b(j) \Phi(t-j)\right|+\left|\sum_{|j|<J_{\varepsilon}} b(j) \Phi(t-j)\right| \\
& <\varepsilon \sum_{|j| \geq J_{\varepsilon}} \frac{C}{(1+|t-j|)^{3}}+\varepsilon\|b\|_{\infty} \sum_{|j|<J_{\varepsilon}} \frac{C}{(1+|t-j|)^{2}} \\
& <C \varepsilon \sum_{j \in \mathbb{Z}} \frac{1}{(1+|t-j|)^{2}} .
\end{aligned}
$$

Since the function $t \in \mathbb{R} \mapsto \sum_{j \in \mathbb{Z}} 1 /(1+|t-j|)^{2}$ has period 1 and is continuous, it is uniformly bounder. This proves the lemma.

Here and in the sequel we set $\prod_{j \in \emptyset} j=1$.

LEMMA 4.5. Let $f$ be a smooth function on $\mathbb{R}$. For every $n=1,2, \ldots$,

$$
\left(\Theta^{n} f\right)(t)=\sum_{j=1}^{n} \theta_{n, j} t^{n+j} f^{(j)}(t) \quad \text { for all } t \in \mathbb{R},
$$

where $\theta_{n, j}=\left(\begin{array}{c}n-1 \\ j-1\end{array}\right) \prod_{\ell=j+1}^{n} \ell$, for every $j=1, \ldots, n$.

Proof. The formula is obviously true when $n=1$. Moreover, $\theta_{n, 1}=n$ ! and, by our conventions, $\theta_{n, n}=1$ for every $n=1,2, \ldots$. Suppose that the formula holds 
when $n=k-1$. Then for every $t$ in $\mathbb{R}$,

$$
\begin{aligned}
\left(\Theta^{k} f\right)(t)= & t^{2}\left(\Theta^{k-1} f\right)^{\prime}(t) \\
= & \sum_{j=1}^{k-1} \theta_{k-1, j}(k-1+j) t^{k+j} f^{(j)}(t)+\theta_{k-1, j} t^{k+j+1} f^{(j+1)}(t) \\
= & \theta_{k, 1} t^{k+1} f^{\prime}(t)+\sum_{j=2}^{k-1}\left(\theta_{k-1, j}(k-1+j)+\theta_{k-1, j-1}\right) t^{n+j} f^{(j)}(t) \\
& +\theta_{k, k} t^{2 k} f^{(k)}(t) .
\end{aligned}
$$

So we only need to prove that for $j=2, \ldots, k-1, \theta_{k, j}=(k-1+j) \theta_{k-1, j}+\theta_{k-1, j-1}$, which follows easily using the identity $\left(\begin{array}{c}k-2 \\ j-2\end{array}\right)=\left(\begin{array}{c}k-1 \\ j-1\end{array}\right)-\left(\begin{array}{c}k-2 \\ j-1\end{array}\right)$.

LEMMA 4.6. Suppose that $p, r, s$ are nonnegative integers and $h=0,1$. Then for every $x$ in $\mathbb{R}$ the sum

$$
\sum_{j \in \mathbb{Z}}(j+h / 2)^{r}(x-(j+h / 2))^{s} \varphi^{(p)}(x-(j+h / 2))
$$

is equal to $(-1)^{s} p !\left(\begin{array}{c}r \\ p-s\end{array}\right) x^{s+r-p}$ if $s \leq p \leq s+r$ and 0 otherwise.

Proof. These identities can easily be derived from the Poisson summation formula (3), where $t=0$ and $\xi=h / 2$, applied to the function $f$, where

$$
f(u)=\left(\frac{d}{d u}\right)^{r}\left[e^{-2 \pi i x u}\left(\frac{d}{d u}\right)^{s}\left(u^{p}\left(\mathscr{F}^{-1} \varphi\right)(u)\right)\right], \quad u \text { in } \mathbb{R} .
$$

Finally, we mention the following properties of binomial coefficients.

LEMMA 4.7. For every $n=1,2, \ldots$, every $p \in \mathbb{N}$ and every $s=0, \ldots, n-1$,

$$
\begin{aligned}
\sum_{q=0}^{p+1}(-1)^{q}\left(\begin{array}{c}
n+p+1 \\
q
\end{array}\right) & =(-1)^{p+1}\left(\begin{array}{c}
n+p \\
p+1
\end{array}\right), \\
\sum_{q=0}^{p+1}(-1)^{q}\left(\begin{array}{c}
n+p+1 \\
q
\end{array}\right)\left(\begin{array}{c}
s+p+1-q \\
s
\end{array}\right) & =(-1)^{p+1}\left(\begin{array}{c}
n+p-s \\
p+1
\end{array}\right) .
\end{aligned}
$$

PROOF. This is routine.

We can now prove the main result of this section, that is, Theorem 4.3. 
Proof. Consider first the case where $n=0$. By equation (4) we can write

$$
\begin{aligned}
\lim _{|t| \rightarrow+\infty} A_{h}(t) & =\gamma_{0}+\lim _{|t| \rightarrow+\infty}\left(\sum_{j \in \mathbb{Z}} a(j) \varphi(t-(j+h / 2))-\gamma_{0} \sum_{j \in \mathbb{Z}} \varphi(t-(j+h / 2))\right) \\
& =\gamma_{0}+\lim _{|t| \rightarrow+\infty} \sum_{j \in \mathbb{Z}}\left(a(j)-\gamma_{0}\right) \varphi(t-(j+h / 2))=\gamma_{0}
\end{aligned}
$$

since $a(j)-\gamma_{0}=o(1)$ when $j \rightarrow \infty$ and by Lemma 4.4. Therefore in the following we may and shall suppose that $\gamma_{0}=0$. Let $n \geq 1$. By Lemma 4.5 we need to evaluate the limit of $\left(\Theta^{n} A_{h}\right)(t)$ as $|t|$ tends to $+\infty$, that is, of the following:

$$
\begin{aligned}
\sum_{p=1}^{n} & \theta_{n, p} \sum_{j \in \mathbb{Z}} a(j)(t-(j+h / 2)+(j+h / 2))^{n+p} \varphi^{(p)}(t-(j+h / 2)) \\
= & \sum_{p=1}^{n} \sum_{q=0}^{n+p} \theta_{n, p}\left(\begin{array}{c}
n+p \\
q
\end{array}\right) \\
& \times \sum_{j \in \mathbb{Z}} a(j)(t-(j+h / 2))^{n+p-q}(j+h / 2)^{q} \varphi^{(p)}(t-(j+h / 2)) .
\end{aligned}
$$

Since the sequence $a=(a(j))_{j \in \mathbb{Z}}$ has an asymptotic expansion, $\left(\Theta^{n} A_{h}\right)(t)$ is equal to

$$
\begin{aligned}
\sum_{p=1}^{n} & \sum_{q=0}^{n+p} \theta_{n, p}\left(\begin{array}{c}
n+p \\
q
\end{array}\right) \sum_{j \in \mathbb{Z}}\left(\sum_{r=0}^{q} \frac{\gamma_{r}}{(j+h / 2)^{r}}+o\left(|j|^{-q}\right)\right) \\
& \times(t-(j+h / 2))^{n+p-q}(j+h / 2)^{q} \varphi^{(p)}(t-(j+h / 2)) \\
= & \sum_{p=1}^{n} \sum_{q=0}^{n+p} \sum_{r=0}^{q} \gamma_{r} \theta_{n, p}\left(\begin{array}{c}
n+p \\
q
\end{array}\right) \\
& \times\left(\sum_{j \in \mathbb{Z}}(t-(j+h / 2))^{n+p-q}(j+h / 2)^{q-r} \varphi^{(p)}(t-(j+h / 2))\right) \\
& +\sum_{p=1}^{n} \sum_{q=0}^{n+p} \theta_{n, p}\left(\begin{array}{c}
n+p \\
q
\end{array}\right)\left(\sum_{j \in \mathbb{Z}} o(1)(t-(j+h / 2))^{n+p-q} \varphi^{(p)}(t-(j+h / 2))\right) \\
= & R_{1}(t)+R_{2}(t) .
\end{aligned}
$$

By Lemma 4.4, the remainder $R_{2}(t)$ tends to zero as $|t| \rightarrow+\infty$. Moreover by Lemma 4.6 we can evaluate the series in $R_{1}(t)$. The sum is 0 unless $1 \leq r \leq n \leq q$, and we may write $R_{1}(t)=\sum_{r=1}^{n} \gamma_{r} t^{n-r} R_{1, r}$. Equation (6) follows, if we show that $R_{1, r}=(-1)^{n} n$ ! when $r=n$ and $R_{1, r}=0$ otherwise. 
Remembering the form of the coefficients $\theta_{n, p}$ in Lemma 4.5, and by Lemma 4.7, we obtain

$$
\begin{aligned}
R_{1, r} & =\sum_{p=1}^{n} \theta_{n, p} p ! \sum_{q=n}^{n+p}(-1)^{n+p-q}\left(\begin{array}{c}
n+p \\
q
\end{array}\right)\left(\begin{array}{c}
q-r \\
n-r
\end{array}\right) \\
& =\sum_{p=0}^{n-1} \theta_{n, p+1}(p+1) ! \sum_{q=0}^{p+1}(-1)^{p+1-q}\left(\begin{array}{c}
n+p+1 \\
n+q
\end{array}\right)\left(\begin{array}{c}
n-r+q \\
n-r
\end{array}\right) \\
& =n ! \sum_{p=0}^{n-1}\left(\begin{array}{c}
n-1 \\
p
\end{array}\right) \sum_{q=0}^{p+1}(-1)^{q}\left(\begin{array}{c}
n+p+1 \\
n+p+1-q
\end{array}\right)\left(\begin{array}{c}
n-r+p+1-q \\
n-r
\end{array}\right) \\
& =n ! \sum_{p=0}^{n-1}\left(\begin{array}{c}
n-1 \\
p
\end{array}\right) \sum_{q=0}^{p+1}(-1)^{q}\left(\begin{array}{c}
n+p+1 \\
q
\end{array}\right)\left(\begin{array}{c}
n-r+p+1-q \\
n-r
\end{array}\right) . \\
& =n ! \sum_{p=0}^{n-1}(-1)^{p+1}\left(\begin{array}{c}
n-1 \\
p
\end{array}\right)\left(\begin{array}{c}
p+r \\
p+1
\end{array}\right) \\
& =-\frac{n !}{(r-1) !} \sum_{p=0}^{n-1}(-1)^{p}\left(\begin{array}{c}
n-1 \\
p
\end{array}\right) P_{r-1}(p),
\end{aligned}
$$

where $P_{r-1}(p)$ is a polynomial in $p$ of degree $r-1$ and leading coefficient 1 . Since for any $n \geq 1$ and $j=0, \ldots, n-1$, we have

$$
\sum_{p=0}^{n-1}(-1)^{p}\left(\begin{array}{c}
n-1 \\
p
\end{array}\right) p^{j}= \begin{cases}0, & \text { if } j=0, \ldots, n-2 \\
(-1)^{n-1}(n-1) !, & \text { if } j=n-1,\end{cases}
$$

we conclude that $R_{1, n}=(-1)^{n} n$ !. Moreover, if $r$ is in $\{1, \ldots, n-1\}$, then $r-1 \leq$ $n-2<n-1$, and $R_{1, r}=0$.

\section{Proof of Theorem 3.1}

In [1] we proved that a function $\psi: \mathbb{R}^{+} \times \mathbb{N} \rightarrow \mathbb{C}$ such that $\psi(\lambda, d)=\lambda^{-\alpha / 2} \psi(1, d)$, for almost every $(\lambda, d)$ in $\mathbb{R}^{+} \times \mathbb{N}$, is the Gelfand transform of a biradial kernel of type $\alpha$ on the $H$-type group $N$ if and only if for every $n, j$ in $\mathbb{N}$, the following limits

$$
\lim _{d \rightarrow \infty} d^{\alpha / 2+n+j}\left[M_{+}^{n} M_{-}^{j} \psi\right](1, d)=(-1)^{j+n} \lim _{d \rightarrow \infty} d^{\alpha / 2+n+j}\left[M_{-}^{n} M_{+}^{j} \psi\right](1, d)
$$

exist and are finite. Note that

$$
\left[M_{ \pm}^{j} \widehat{K}\right](1, d)=(-1)^{j}\left[\prod_{p=0}^{j-1}\left(p+\mathscr{D}_{ \pm}\right) \widehat{K}\right](1, d) .
$$


LEMMA 5.1. Let $K$ be a biradial kernel of type 0. Then there exist complex numbers $c_{j}, j=0,1,2, \ldots$, such that for every $M \geq 0$

$$
\widehat{K}(1, d)=\sum_{j=0}^{M} c_{j}(2 d+m)^{-j}+o\left(d^{-M}\right), \quad d \rightarrow+\infty .
$$

Proof. By Theorem 4.2 all we need to prove is that the $\operatorname{limits}_{d \rightarrow+\infty} \lim _{n} \widehat{K}(1, d)$ exist and are finite. Clearly $T_{0} \widehat{K}(1, d)=\widehat{K}(1, d)$, so the case where $n=0$ is straightforward.

We prove that for any positive integer $n$

$$
T_{n} \widehat{K}(1, d)=\sum_{j=1}^{n} P_{j}(d)\left[M_{-}^{j} \widehat{K}\right](1, d+n),
$$

where $P_{j}$ is a polynomial of degree at most $j$. From this the lemma follows.

The case where $n=1$ is easy, since

$$
T_{1} \widehat{K}(1, d)=d(d+1)(\widehat{K}(1, d+1)-\widehat{K}(1, d))=-d M_{-} \widehat{K}(1, d+1) .
$$

Let us suppose equation (11) holds when $n=k-1$. By the discrete Leibniz rule (1), when $n=k$ we obtain that $T_{k} \widehat{K}(1, d)$ equals

$$
\begin{aligned}
\frac{d(d+k)}{k} \Delta T_{k-1} \widehat{K}(1, d)= & \frac{d(d+k)}{k} \sum_{j=1}^{k-1} \Delta P_{j}(d) M_{-}^{j} \widehat{K}(1, d+k) \\
& +P_{j}(d) \Delta M_{-}^{j} \widehat{K}(1, d+k-1) .
\end{aligned}
$$

Note that

$$
\begin{aligned}
\Delta M_{-}^{j} \widehat{K}(1, d+k-1) & =\frac{1}{d+k} \mathscr{D}_{-} M_{-}^{j} \widehat{K}(1, d+k) \\
& =\frac{1}{d+k}\left(-j+j+\mathscr{D}_{-}\right) M_{-}^{j} \widehat{K}(1, d+k) \\
& =-\frac{1}{d+k} M_{-}^{j+1} \widehat{K}(1, d+k)-\frac{j}{d+k} M_{-}^{j} \widehat{K}(1, d+k) .
\end{aligned}
$$

Therefore $T_{k} \widehat{K}(1, d)$ is equal to

$$
\begin{gathered}
\frac{d}{k} \sum_{j=1}^{k-1}\left(-P_{j}(d) M_{-}^{j+1} \widehat{K}(1, d+k)+\left((d+k) \Delta P_{j}(d)-j P_{j}(d)\right)\right) M_{-}^{j} \widehat{K}(1, d+k) \\
=\left(\sum_{j=1}^{k-1} P_{j+1}(d) M_{-}^{j+1}+d\left[(d+k) \Delta P_{j}(d)-j P_{j}(d)\right] M_{-}^{j}\right) \widehat{K}(1, d+k) .
\end{gathered}
$$


Let $P_{j}(d)=a_{j} d^{j}+Q_{j-1}(d)$ for some polynomial $Q_{j-1}$ of degree $j-1$. It is easy to check that $\Delta P_{j}(d)=j a_{j} d^{j-1}+\Delta Q_{j-1}(d)$, therefore the degree of the polynomial $(d+k) \Delta P_{j}(d)-j P_{j}(d)$ is at most $j-1$.

In the following proposition we prove that $c_{2 j+1}=0, j=0,1,2, \ldots$, where $c_{j}$ are the coefficients defined in Lemma 5.1.

PROPOSITION 5.2. Suppose that $K$ is a biradial kernel of type 0 . Then there exist complex numbers $c_{2 j}, j=0,1,2, \ldots$, such that for every $M \geq 0$

$$
\widehat{K}(1, d)=\sum_{j=0}^{M} c_{2 j}(2 d+m)^{-2 j}+o\left(d^{-2 M}\right), \quad d \rightarrow+\infty .
$$

Proof. Using the notation of Lemma 5.1 we shall prove that $c_{j}=(-1)^{j} c_{j}$.

By equation (9), where $n=0$, and by (10), we obtain

$$
\begin{aligned}
& \lim _{d \rightarrow+\infty} d^{j}\left[\prod_{p=0}^{j-1}\left(p+\mathscr{D}_{+}\right) \widehat{K}\right](1, d) \\
& =(-1)^{j} \lim _{d \rightarrow+\infty} d^{j}\left[\prod_{p=0}^{j-1}\left(p+\mathscr{D}_{-}\right) \widehat{K}\right](1, d) .
\end{aligned}
$$

For every $q$ in $\mathbb{N}$, let $g_{q}$ be the sequence defined by $g_{q}(d)=(2 d+m)^{-q}$ for all $d \in \mathbb{N}$, and let $\left(a_{q}\right)$ be a sequence of complex numbers. We claim that, when $1 \leq j \leq M$,

$$
\left[\prod_{p=0}^{j-1}\left(p+\mathscr{D}_{ \pm}\right)\right]\left(\sum_{q=1}^{M} a_{q} g_{q}\right)=\sum_{q=j}^{M} \eta_{q, j}^{ \pm} g_{q}+o\left(d^{-M}\right),
$$

where $\eta_{1,1}^{ \pm}=-a_{1}$ and, if $1 \leq j \leq q$, there exist rational numbers $\mu_{p, q, j}$, depending only on $p, q, j$, such that

$$
\eta_{q, j}^{ \pm}=(-1)^{j} \frac{q !}{(q-j) !} a_{q}+\sum_{p=1}^{q-1}( \pm 1)^{p-q} \mu_{p, q, j} a_{p} .
$$

If this holds, we may conclude that $\lim _{d \rightarrow+\infty} d \mathscr{D}_{ \pm} \widehat{K}(1, d)=-c_{1} / 2$ and, when $j>1$,

$$
\begin{aligned}
\lim _{d \rightarrow+\infty} d^{j} & {\left[\prod_{p=0}^{j-1}\left(p+\mathscr{D}_{ \pm}\right) \widehat{K}\right](1, d) } \\
\quad= & \lim _{d \rightarrow+\infty} d^{j}\left\{\left[\prod_{p=0}^{j-1}\left(p+\mathscr{D}_{ \pm}\right)\left(\sum_{q=1}^{j} c_{q} g_{q}\right)\right](d)+o\left(d^{-j}\right)\right\}
\end{aligned}
$$




$$
=\eta_{j, j}^{ \pm} 2^{-j}=2^{-j}\left((-1)^{j} j ! c_{j}+\sum_{p=1}^{j-1}( \pm 1)^{p-j} \mu_{p, j, j} c_{p}\right) .
$$

By (12), we obtain $c_{1}=-c_{1}$ and

$$
(-1)^{j} j ! c_{j}+\sum_{p=1}^{j-1} \mu_{p, j, j} c_{p}=j ! c_{j}+\sum_{p=1}^{j-1} \mu_{p, j, j}(-1)^{p} c_{p} \quad \text { for all } j>1 .
$$

By induction on $j$ it follows easily that $c_{j}=(-1)^{j} c_{j}$ for every $j$ in $\mathbb{N}$.

We now prove the claim, that is, equation (13). It is easy to check that

$$
\mathscr{D}_{ \pm} g_{n}(d)=-n g_{n}(d)+\sum_{p=n+1}^{\infty}( \pm 1)^{p-n} \beta_{n, p} g_{p}(d)
$$

Moreover, when $M>n \geq 1$ and $d \rightarrow+\infty$, we have

$$
\begin{aligned}
\mathscr{D}^{ \pm}\left(\sum_{q=n}^{M} a_{q} g_{q}\right)(d)= & -n a_{n} g_{n}(d) \\
& +\sum_{q=n+1}^{M}\left(-q a_{q}+\sum_{p=n}^{q-1}( \pm 1)^{q-p} \beta_{p, q} a_{p}\right) g_{q}(d)+o\left(d^{-M}\right),
\end{aligned}
$$

where

$$
\beta_{p, q}=2^{q-p}\left[\frac{m}{2}\left(\begin{array}{c}
-p \\
q-p
\end{array}\right)+\left(\begin{array}{c}
-p \\
q-p+1
\end{array}\right)\right] .
$$

We shall prove the claim by induction on $j$. Suppose that $j=1$. By (14), we obtain

$$
\mathscr{D}^{ \pm}\left(\sum_{q=1}^{M} a_{q} g_{q}\right)(d)=\sum_{q=1}^{M} \eta_{q, 1}^{ \pm} g_{q}(d)+o\left(d^{-M}\right)
$$

where $\eta_{1,1}^{ \pm}=-a_{1}$ and, when $q>1$,

$$
\eta_{q, 1}^{ \pm}=-q a_{q}+\sum_{p=1}^{q-1}( \pm 1)^{q-p} \beta_{p, q} a_{p}
$$

Suppose that the thesis holds when $j=k-1$. Then when $j=k$, by (14), we obtain

$$
\begin{aligned}
{\left[\prod_{p=0}^{k-1}\left(p+\mathscr{D}_{ \pm}\right)\right]\left(\sum_{q=1}^{M} a_{q} g_{q}\right) } & =\left(k-1+\mathscr{D}_{ \pm}\right)\left(\sum_{q=k-1}^{M} \eta_{q, k-1}^{ \pm} g_{q}+o\left(d^{-M}\right)\right) \\
& =\sum_{q=k}^{M} \eta_{q, k}^{ \pm} g_{q}+o\left(d^{-M}\right),
\end{aligned}
$$


where

$$
\begin{aligned}
\eta_{q, k}^{ \pm}= & (k-1-q) \eta_{q, k-1}^{ \pm}+\sum_{p=k-1}^{q-1}( \pm 1)^{q-p} \beta_{p, q} \eta_{p, k-1}^{ \pm} \\
= & (k-1-q)\left((-1)^{k-1} \frac{q !}{(q-k+1) !} a_{q}+\sum_{p=1}^{q-1}( \pm 1)^{p-q} \mu_{p, q, k-1} a_{p}\right) \\
& +\sum_{p=k-1}^{q-1}( \pm 1)^{q-p} \beta_{p, q}\left(\sum_{i=1}^{p-1}( \pm 1)^{i-p} \mu_{i, p, k-1} a_{i}+(-1)^{k-1} \frac{p !}{(p-k+1) !} a_{p}\right) \\
= & (-1)^{k} \frac{q !}{(q-k) !} a_{q}+\sum_{p=1}^{q-1}( \pm 1)^{p-q} \mu_{p, q, k} a_{p} .
\end{aligned}
$$

The proof is now complete.

COROLLARY 5.3. Let $\alpha$ be in $[0, Q)$ and suppose that $K$ is a biradial kernel of type $\alpha$. Then there exist complex numbers $c_{2 j}, j=0,1,2, \ldots$, such that for every $M \geq 0$

$$
\widehat{K}(1, d)=\sum_{j=0}^{M} c_{2 j}(2 d+m)^{-2 j-\alpha / 2}+o\left(d^{-2 M-\alpha / 2}\right), \quad d \rightarrow+\infty .
$$

Proof. Suppose that $K$ is a biradial kernel of type $\alpha$. Then $\mathscr{L}_{1}^{\alpha / 2} K$ is a biradial kernel of type 0 and for almost every $(\lambda, d)$ in $\mathbb{R}^{+} \times \mathbb{N}$

$$
\widehat{K}(\lambda, d)=\widehat{\mathscr{L}_{1}^{\alpha / 2} K}(\lambda, d)[\lambda(2 d+m)]^{-\alpha / 2} .
$$

The thesis follows from Proposition 5.2.

\section{Proof of Theorem 3.2 in the Heisenberg group case}

In this section we prove the analogue of Theorem 3.2 in the case where the homogeneous distributions are radial and $k=1$, that is, $N$ is the Heisenberg group of real dimension $2 m+1$; the proof of Theorem 3.2 for biradial kernels on Heisenberg type groups is analogous (see the remark at the end of this section). We recall that a function $f$ on $N$ is said to be radial if there exists a function $f_{0}$ on $\mathbb{R} \times \mathfrak{z}$ such that $f(X, Z)=f_{0}(|X|, Z)$ for every $(X, Z)$ in $N$.

We denote by $\mathbb{A}^{m}$ the Heisenberg group of real dimension $2 m+1$. We refer to [2] for details on spherical functions on the Heisenberg group $\mathbb{H}^{m}$ that are related to the Gelfand pair $\left(\mathbb{H}^{m}, \mathrm{U}(m)\right)$. We only mention that in this case they are parametrized 
by a pair $(\lambda, d)$, where $\lambda$ is in $\mathbb{R}^{*}$ and $d$ in $\mathbb{N}$, and by a nonnegative real number $\xi$. If we identify the pair $(\lambda, d)$ with the point $(\lambda,|\lambda|(2 d+m))$ in $\mathbb{R}^{2}$ and $\xi$ with the point $(0, \xi)$ and use the Euclidean topology in $\mathbb{R}^{2}$, then we obtain the compact open topology on the Gelfand spectrum. What we prove for radial kernels of type $\alpha$ is the following theorem.

THEOREM 6.1. Suppose that $\alpha$ is in $[0, Q)$ and $\psi: \mathbb{R}^{*} \times \mathbb{N} \rightarrow \mathbb{C}$. The following conditions are equivalent:

(a') the function $\psi$ is the Gelfand transform of a radial kernel of type $\alpha$ on $\mathbb{H}^{m}$;

(c') there exists a smooth function $F$ on $\mathbb{R}_{+}^{2}$, homogeneous of degree $-\alpha / 2$ such that $F(\lambda,|\lambda|(2 d+m))=\psi(\lambda, d)$ for almost every $(\lambda, d)$ in $\mathbb{R}^{*} \times \mathbb{N}$.

We first prove that $\left(\mathrm{a}^{\prime}\right)$ implies $\left(\mathrm{c}^{\prime}\right)$ when $\alpha=0$, using Geller's asymptotic expansion [8]: $\psi$ is the Gelfand transform of a radial kernel of type 0 on $\mathbb{H}^{m}$ if and only if

$$
\psi(\lambda, d)=\psi(\lambda /|\lambda|, d)=\sum_{j=0}^{M}(\lambda /|\lambda|)^{j} c_{j}(2 d+m)^{-j}+o\left(d^{-M}\right), \quad d \rightarrow+\infty .
$$

The result for kernels of type 0 will follow from Proposition 6.2. Then we will extend this result to radial kernels of type $\alpha$ in Corollary 6.3.

The proof of $\left(c^{\prime}\right)$ implies $\left(a^{\prime}\right)$ is standard and outlined in Proposition 6.4.

PROPOSITION 6.2. Let $\psi: \mathbb{R}^{*} \times \mathbb{N} \rightarrow \mathbb{C}$ and suppose that $\psi(\lambda, d)=\psi(\lambda /|\lambda|, d)$ for almost every $(\lambda, d)$ in $\mathbb{R}^{*} \times \mathbb{N}$. If there exist complex numbers $c_{j}, j=0,1,2, \ldots$, such that

$$
\psi( \pm 1, d)=\sum_{j=0}^{M}( \pm 1)^{j} c_{j}(2 d+m)^{-j}+o\left(d^{-M}\right), \quad d \rightarrow+\infty,
$$

then there exists a smooth function $F$ on $\mathbb{R}_{+}^{2}$, homogeneous of degree 0 , such that $F(\lambda,|\lambda|(2 d+m))=\psi(\lambda, d)$ for almost every $(\lambda, d) \in \mathbb{R}^{*} \times \mathbb{N}$.

PROOF. First we extend the definition of $\psi(\lambda, j)$ to negative integers $j$ by letting

$$
\psi(\lambda, j)= \begin{cases}\psi(-\lambda,-j-m) & \text { for all } \lambda \in \mathbb{R}^{+}, j \leq-m, \\ 0 & \text { for all } \lambda \in \mathbb{R}^{+}, j \in\{-1,-2, \ldots,-m+1\} .\end{cases}
$$

We define $F: \mathbb{R}_{+}^{2} \rightarrow \mathbb{C}$ by the rule

$$
F(\lambda, \xi)= \begin{cases}\sum_{j \in \mathbb{Z}} \psi(\lambda, j) \varphi\left(\frac{1}{2}\left(\frac{\xi}{|\lambda|}-m\right)-j\right) & \text { for all }(\lambda, \xi) \in \mathbb{R}^{*} \times \mathbb{B}^{+}, \\ c_{0} & \text { for all }(\lambda, \xi) \in\{0\} \times \mathbb{R}^{+},\end{cases}
$$


where $\varphi$ is as in Section 4.2. By the properties (4) of the function $\varphi$

$$
F(\lambda,|\lambda|(2 d+m))=\psi(\lambda, d), \quad \text { for all } d \in \mathbb{N}, \text { and } \lambda \in \mathbb{R}^{+} .
$$

Moreover, it is easy to see that $F$ is in $C^{\infty}\left(\mathbb{R}^{+} \times \mathbb{R}^{+}\right)$and homogeneous of degree 0 . To apply the results of Section 4 , we introduce the following notation. Let $h=m$ (mod 2) and define the two-sided sequences $a_{h}^{ \pm}$by the rules

$$
a_{h}^{+}(j)=\psi\left(1, j-\frac{m-h}{2}\right) \text { and } a_{h}^{-}(j)=\psi\left(-1, j-\frac{m-h}{2}\right)
$$

for all $j \in \mathbb{Z}$. The way we have extended the definition of $\psi(\lambda, j)$ to negative integers $j$ implies that the sequences $a_{h}^{ \pm}$have asymptotic expansions of the form

$$
a_{h}^{ \pm}(j)=\sum_{n=0}^{M}( \pm 1)^{n} \frac{2^{-n} c_{n}}{(j+h / 2)^{n}}+o\left(j^{-M}\right), \quad|j| \rightarrow+\infty .
$$

As in Section 4, let $A_{h}^{ \pm}$denote the function

$$
A_{h}^{ \pm}(t)=\sum_{j \in \mathbb{Z}} a_{h}^{ \pm}(j) \varphi(t-(j+h / 2)) \quad \text { for all } t \in \mathbb{R} .
$$

Then for every $\xi>0$,

$$
F(\lambda, \xi)= \begin{cases}A_{h}^{+}(\xi / 2 \lambda) & \text { if } \lambda>0 \\ c_{0} & \text { if } \lambda=0 \\ A_{h}^{-}(-\xi / 2 \lambda) & \text { if } \lambda<0\end{cases}
$$

By Theorem 4.3, the functions $A_{h}^{ \pm}$are $C^{\infty}(\mathbb{R})$ and

$$
\lim _{t \rightarrow+\infty}\left(\Theta^{n} A_{h}^{ \pm}\right)(t)=(\mp 1)^{n} n ! 2^{-n} c_{n} \quad \text { for all } n \in \mathbb{N},
$$

where, as before, $\Theta$ denotes the differential operator defined by

$$
\Theta f(t)=t^{2} f^{\prime}(t) \text { for all } t \in \mathbb{R} \text { and } f \in C^{\infty}(\mathbb{R}) .
$$

If $\check{f}$ is defined by $\check{f}(t)=f(1 / t)$, for every $t$ in $\mathbb{R}^{*}$, then $(\check{f})^{(n)}(t)=(-1)^{n}\left(\Theta^{n} f\right)(1 / t)$ for every $t$ in $\mathbb{R}^{*}$.

For the sake of brevity, for every $n$ in $\mathbb{N}$, denote by $\Xi_{n}$ the operator acting on smooth functions $f$ on $\mathbb{R}$ by $\Xi_{n} f(t)=t^{n} f^{(n)}(t)$ for all $t \in \mathbb{R}$. From Lemma 4.5 , it follows that for every smooth function $f$ on $\mathbb{R}$ and $j \geq 1$,

$$
\left(\Xi_{j} f\right)(t)=(-1)^{j} t^{j}\left(\Theta^{j} \check{f}\right)\left(\frac{1}{t}\right)=\sum_{s=1}^{j}(-1)^{j+s} \theta_{j, s} t^{-s}\left(\Theta^{s} f\right)(t) \text { for all } t \in \mathbb{R}^{*} \text {. }
$$


Therefore, by (15), for every $n$ in $\mathbb{N}$ and $j \geq 1$,

$$
\lim _{|x| \rightarrow+\infty} \Xi_{j}\left(\Theta^{n} A_{h}^{ \pm}\right)(t)=\lim _{|t| \rightarrow+\infty} \sum_{s=1}^{j}(-1)^{j+s} \theta_{j, s} t^{-s}\left(\Theta^{n+s} A_{h}^{ \pm}\right)(t)=0 .
$$

We now prove that $F$ is smooth on the positive $\xi$-axis. First of all, using (15) where $n=0$, it is easy to prove that $F$ is continuous. Straightforward computations show that

$$
\partial_{\xi} F(\lambda, \xi)= \begin{cases}(1 / \xi)\left(\Xi_{1} A_{h}^{+}\right)(\xi / 2 \lambda) & \text { if } \lambda>0 \\ 0 & \text { if } \lambda=0 \\ (1 / \xi)\left(\Xi_{1} A_{h}^{-}\right)(-\xi / 2 \lambda) & \text { if } \lambda<0\end{cases}
$$

This partial derivative is continuous, because if $(\lambda, \xi) \rightarrow\left(0, \xi_{0}\right)$ in $\mathbb{R}_{+}^{2}$, then $|t|=|\xi / 2 \lambda| \rightarrow+\infty$ and one can use (16) where $n=0$ and $j=1$. As for the derivative in the $\lambda$-direction, one can easily evaluate

$$
\partial_{\lambda} F(\lambda, \xi)= \begin{cases}-(2 / \xi)\left(\Theta A_{h}^{+}\right)(\xi / 2 \lambda) & \text { if } \lambda>0 \\ (2 / \xi)\left(\Theta A_{h}^{-}\right)(-\xi / 2 \lambda) & \text { if } \lambda<0\end{cases}
$$

Moreover, by (15),

$$
\lim _{\lambda \rightarrow 0^{ \pm}} \partial_{\lambda} F(\lambda, \xi)=\mp \frac{2}{\xi} \lim _{t \rightarrow+\infty}\left(\Theta A_{h}^{ \pm}\right)(t)=-\frac{1}{\xi} c_{1} .
$$

Therefore $F$ has continuous partial derivatives.

When $\beta=\left(\beta_{1}, \ldots, \beta_{2 s}\right)$ is a multi-index in $\mathbb{N}^{2 s}$ of length $r$, we denote by $\partial^{\beta}$ the operator $\partial_{\lambda}^{\beta_{1}} \partial_{\xi}^{\beta_{2}} \cdots \partial_{\lambda}^{\beta_{2 s-1}} \partial_{\xi}^{\beta_{2 s}}$. In a similar way, using (15) and (16), one can prove by induction on $r$ that, if $\sum_{j=0}^{s-1} \beta_{2 j+1}=n$ and $p=r-n$, then when $\xi>0$ the derivative $\partial^{\beta} F(\lambda, \xi)$ equals

$$
\begin{cases}\frac{(-2)^{n}}{\xi^{n+p}} \sum_{j=0}^{p}\left(\begin{array}{l}
p \\
j
\end{array}\right)(-n) \cdots(-n-p+j+1)\left[\Xi_{j} \Theta^{n} A_{h}^{+}\right]\left(\frac{\xi}{2 \lambda}\right) & \text { if } \lambda>0, \\
\frac{2^{n}}{\xi^{n+p}} \sum_{j=0}^{p}\left(\begin{array}{l}
p \\
j
\end{array}\right)(-n) \cdots(-n-p+j+1)\left[\Xi_{j} \Theta^{n} A_{h}^{-}\right]\left(-\frac{\xi}{2 \lambda}\right) & \text { if } \lambda<0,\end{cases}
$$

and $\partial^{\beta} F$ is continuous with $\partial^{\beta} F(0, \xi)=n ! c_{n}(-n) \cdots(-n-p+1) / \xi^{n+p}$.

COROLlaRY 6.3. Let $\alpha$ be in $[0, Q)$ and suppose that $\psi: \mathbb{R}^{*} \times \mathbb{N} \rightarrow \mathbb{C}$ satisfies $\psi(\lambda, d)=|\lambda|^{-\alpha / 2} \psi(\lambda /|\lambda|, d)$ for almost every $(\lambda, d)$ in $\mathbb{R}^{*} \times \mathbb{N}$. If there exist complex numbers $c_{j}, j=0,1,2, \ldots$, such that

$$
\psi( \pm 1, d)=\sum_{j=0}^{M}( \pm 1)^{j} c_{j}(2 d+m)^{-j-\alpha / 2}+o\left(d^{-M-\alpha / 2}\right), \quad d \rightarrow+\infty,
$$


then there exists a smooth function $F$ on $\mathbb{R}_{+}^{2}$, homogeneous of degree $-\alpha / 2$, such that $F(\lambda,|\lambda|(2 d+m))=\psi(\lambda, d)$ for almost every $(\lambda, d) \in \mathbb{R}^{*} \times \mathbb{N}$.

Proof. The function $\psi_{0}: \mathbb{R}^{*} \times \mathbb{N} \rightarrow \mathbb{C}$ defined by

$$
\psi_{0}(\lambda, d)=(|\lambda|(2 d+m))^{\alpha / 2} \psi(\lambda, d) \text { for all }(\lambda, d) \in \mathbb{R}^{*} \times \mathbb{N}
$$

satisfies the hypothesis of Proposition 6.2. Therefore, there exists a smooth function $H$ on $\mathbb{R}_{+}^{2}$, homogeneous of degree 0 such that $H(\lambda,|\lambda|(2 d+m))=\psi_{0}(\lambda, d)$ for almost every $(\lambda, d)$ in $\mathbb{R}^{*} \times \mathbb{N}$. We define $F(\lambda, \xi)=H(\lambda, \xi) \xi^{-\alpha / 2}$ for all $(\lambda, \xi) \in \mathbb{R}_{+}^{2}$. The function $F$ satisfies the required conditions.

PRoposition 6.4. Let $\alpha$ be in $[0, Q)$. If $F: \mathbb{R}_{+}^{2} \rightarrow \mathbb{C}$ is a smooth and homogeneous function of degree $-\alpha / 2$, then there exists a radial kernel $K$ of type $\alpha$ on $\mathrm{Bt}^{\mathrm{m}}$ such that $F(\lambda,|\lambda|(2 d+m))=\widehat{K}(\lambda, d)$ for almost every $(\lambda, d)$ in $\mathbb{B}^{*} \times \mathbb{N}$.

PROOF. As in [13, page 242] we fix a function $\Phi$ in $C_{c}^{\infty}(\mathbb{R})$, with support in $[1 / 2,4]$ and such that $\sum_{j=-\infty}^{+\infty} \Phi\left(2^{j} \xi\right)=1$ for all $\xi>0$. We define the radial functions $K_{j}$ : $\mathbb{H}^{m} \rightarrow \mathbb{C}, j$ in $\mathbb{Z}$, by the rules

$$
\begin{aligned}
& \widehat{K}_{0}(\lambda, d)=F(\lambda,|\lambda|(2 d+m)) \Phi(|\lambda|(2 d+m)), \\
& \widehat{K}_{j}(\lambda, d)=2^{j \alpha / 2} \widehat{K}_{0}\left(2^{j} \lambda, d\right)=\Phi\left(2^{j}|\lambda|(2 d+m)\right) F(\lambda,|\lambda|(2 d+m))
\end{aligned}
$$

for all $(\lambda, d) \in \mathbb{R}^{*} \times \mathbb{N}$. As in [1, Lemma 7.1] one can prove that the series $\sum_{j=-\infty}^{+\infty} K_{j}$ converges in $\mathscr{S}^{\prime}$ to a biradial kernel $K$ of type $\alpha$. Finally, $F(\lambda,|\lambda|(2 d+m))=\widehat{K}(\lambda, d)$ for almost every $(\lambda, d) \in \mathbb{R}^{*} \times \mathbb{N}$, as required.

REMARK. In the case of biradial kernels $K$ on Heisenberg type groups $N$, the function $\psi=\widehat{K}$ is initially defined in $\mathbb{R}^{+} \times \mathbb{N}$. We extend it to obtain an even function on $\mathbb{R}^{*} \times \mathbb{N}$. Moreover, remember that in the asymptotic expansion we have $c_{2 j+1}=0, j=0,1,2, \ldots$ by Proposition 5.2. The proofs of Proposition 6.2 and Corollary 6.3 then show that $F$ is an even smooth function on $\mathbb{R}_{+}^{2}$, as stated in Theorem 3.2. Analogously, Proposition 6.4 adapts to biradial kernels on Heisenberg type groups by requiring that the function $F$ is even.

\section{References}

[1] F. Astengo and B. Di Blasio, 'The Schwartz space and homogeneous distributions on $H$-type groups', Monatsh. Math. 132 (2001), 197-214. 
[2] C. Benson, J. Jenkins and G. Ratcliff, 'The spherical transform of a Schwartz function on the Heisenberg group', J. Funct. Anal. 154 (1998), 379-423.

[3] M. Cowling, 'Unitary and uniformly bounded representations of some simple Lie groups', in: Harmonic analysis and group representations, C.I.M.E. (Liguori, Napoli, 1982) pp. 49-128.

[4] G. B. Folland, 'Subelliptic estimates and function spaces on nilpotent Lie groups', Ark. Mat. 13 (1975), 161-207.

[5] G. B. Folland and E. M. Stein, 'Estimates for the $\overrightarrow{\partial_{b}}$ complex and analysis on the Heisenberg group', Comm. Pure Appl. Math. 27 (1974), 429-522.

[6] A. J. Fraser, 'Convolution kernels of $(n+1)$-fold Marcinkiewicz multipliers on the Heisenberg group', Bull. Austral. Math. Soc. 64 (2001), 353-376.

[7] _ 'An $(n+1)$-fold Marcinkiewicz multiplier theorem on the Heisenberg group', Bull. Austral. Math. Soc. 63 (2001), 35-58.

[8] D. Geller, 'Local solvability and homogeneous distributions on the Heisenberg group', Comm. Partial Differential Equations 5 (1980), 475-560.

[9] A. Kaplan, 'Fundamental solution for a class of hypoelliptic PDE generated by composition of quadratic forms', Trans. Amer. Math. Soc. 258 (1980), 147-153.

[10] D. Müller, M. Peloso and F. Ricci, ' $L$-spectral multipliers for the Hodge Laplacian acting on 1-forms on the Heisenberg group', preprint.

[11] D. Müller, F. Ricci and E. M. Stein, 'Marcinkiewicz multipliers and multi-parameter structure on Heisenberg (-type) groups, I', Invent. Math. 119 (1995), 199-233.

[12] S. E. Slome, 'The heisenberg group and the group Fourier transform of regular homogeneous distributions', Studia Math. 143 (2000), 251-266.

[13] E. M. Stein, Harmonic analysis real variable methods, orthogonality, and oscillatory integrals (Princeton University Press, Princeton, 1993).

Dipartimento di Matematica

Università di Genova

16.146 Genova

Italia

e-mail: astengo@dima.unige.it
Dipartimento di Matematica Università di Roma "Tor Vergata" 00133 Roma Italia e-mail:diblasio@mat.uniroma2.it 